JOURNAL OF THE

AMERICAN MATHEMATICAL SOCIETY

Volume 25, Number 2, April 2012, Pages 507-531

S 0894-0347(2011)00718-2

Article electronically published on October 6, 2011

\title{
BURKHOLDER INTEGRALS, MORREY'S PROBLEM AND QUASICONFORMAL MAPPINGS
}

\author{
KARI ASTALA, TADEUSZ IWANIEC, ISTVÁN PRAUSE, AND EERO SAKSMAN
}

\section{INTRODUCTION}

A continuous function $\mathbf{E}: \mathbb{R}^{n \times n} \rightarrow \mathbb{R}$ is said to be quasiconvex if for every $f \in A+\mathscr{C}_{\circ}^{\infty}\left(\Omega, \mathbb{R}^{n}\right)$ we have

$$
\mathscr{E}[f]:=\int_{\Omega} \mathbf{E}(D f) \mathrm{d} x \geqslant \int_{\Omega} \mathbf{E}(A) \mathrm{d} x=\mathbf{E}(A)|\Omega|,
$$

where $A$ stands for an arbitrary linear mapping (or its matrix) and $\Omega \subset \mathbb{R}^{n}$ is any bounded domain. In other words, one requires that compactly supported perturbations of linear maps do not decrease the value of the integral. This notion is of fundamental importance in the calculus of variations as it is known to characterize lower semicontinuous integrals 37. A weaker notion is that of rank-one convexity, which requires just that $t \mapsto \mathbf{E}(A+t X)$ is convex for any fixed matrix $A$ and for any rank one matrix $X$. Rank-one convexity of an integrand is a local condition and thus much easier to verify than quasiconvexity. That quasiconvexity implies rank-one convexity was known after Morrey's fundamental work in the 1950s, but one had to wait until Šverák's paper [46] to find out that the converse is not true.

However, Šverák's example works only in dimensions $n \geqslant 3$, 43. This leaves the possibility for a different outcome in dimension 2; see [26, [40] for evidence in this direction. Morrey himself was not quite definite in which direction he thought things should be true; see [37, [38, and [11, Sect. 9]. We reveal our own thoughts on the matter by recalling the following conjecture in the spirit of Morrey:

Conjecture 1.1. Rank-one convex functions $\boldsymbol{E}: \mathbb{R}^{2 \times 2} \rightarrow \mathbb{R}$ are quasiconvex.

One says that $\mathbf{E}$ is rank-one concave (resp. quasiconcave) if $\mathbf{- E}$ is rank-one convex (resp. quasiconvex), and null-Lagrangian if both quasiconvex and quasiconcave. In the sequel we will rather discuss concavity, as this turns out to be natural for our methods. The most famous (and, arguably, the most important) rank-one concave function in two dimensions is the Burkholder functional from [23], defined for any $2 \times 2$ matrix $A$ by

$$
\mathbf{B}_{p}(A)=\left(\frac{p}{2} \operatorname{det} A+\left(1-\frac{p}{2}\right)|A|^{2}\right) \cdot|A|^{p-2}, \quad p \geqslant 2 .
$$

Above, we have chosen the normalization $\mathbf{B}_{p}(\mathrm{Id})=1$ with the identity matrix, and the absolute value notation is reserved for the operator norm. It is well known

Received by the editors December 5, 2010 and, in revised form, August 19, 2011.

2010 Mathematics Subject Classification. Primary 30C62, 30C70, 49K10, 49K30.

Key words and phrases. Rank-one convex and quasiconvex variational integrals, critical Sobolev exponents, extremal quasiconformal mappings, Jacobian inequalities. 
that among the wealth of important results the quasiconcavity of the Burkholder functional would imply e.g. the Iwaniec conjecture on the $p$-norm of the BeurlingAhlfors operator; see Conjecture 3.1

The purpose of the present paper is to validate Conjecture 1.1 in an important special case, namely for the above Burkholder functional, in the case of nonnegative integrands, and for perturbations of the identity map:

Theorem 1.2. Let $\Omega \subset \mathbb{R}^{2}$ be a bounded domain and denote by $\operatorname{Id}: \Omega \rightarrow \mathbb{R}^{2}$ the identity map. Assume that $f \in \operatorname{Id}+\mathscr{C}_{\circ}^{\infty}(\Omega)$ satisfies $\boldsymbol{B}_{p}(D f(x)) \geqslant 0$ for $x \in \Omega$. Then

$$
\int_{\Omega} \boldsymbol{B}_{p}(D f) \mathrm{d} x \leqslant \int_{\Omega} \boldsymbol{B}_{p}(\mathrm{Id}) \mathrm{d} x=|\Omega|, \quad p \geqslant 2,
$$

or, written explicitly,

$$
\int_{\Omega}\left(\frac{p}{2} J(z, f)+\left(1-\frac{p}{2}\right)|D f|^{2}\right) \cdot|D f|^{p-2} \leqslant|\Omega| .
$$

Our proof of the above result is based on holomorphic deformations and quasiconformal methods, and we next explain some of the relations between the Burkholder integrals and these maps. The reader is referred to Section 3 for the needed notation. One observes that the condition $\mathbf{B}_{p}(D f) \geqslant 0$ is equivalent to $|D f|^{2} \leqslant \frac{p}{p-2} J_{f}$, which actually amounts to quasiconformality of $f$ (see Section 3). In this setting our result reads as follows:

Theorem 1.3. Let $f: \Omega \longrightarrow \Omega$ be a $K$-quasiconformal map of a bounded open set $\Omega \subset \mathbb{C}$ onto itself, extending continuously up to the boundary, where it coincides with the identity map $\operatorname{Id}(z) \equiv z$. Then

$$
\int_{\Omega} \boldsymbol{B}_{p}(D f) \mathrm{d} x \leqslant \int_{\Omega} \boldsymbol{B}_{p}(\mathrm{Id}) \mathrm{d} x=|\Omega|, \quad \text { for all } 2 \leqslant p \leqslant \frac{2 K}{K-1} .
$$

Further, the equality occurs for a class of (expanding) piecewise radial mappings discussed in Section 5 .

This result says, roughly, that the Burkholder functional is quasiconcave within quasiconformal perturbations of the identity. It is quite interesting that there is an equality in the above theorem for a large class of radial-like maps. When smooth and $p<2 K /(K-1)$, these are all local maxima for the functional; see Proposition 5.2 and Corollary 5.3 for details. In particular, the identity map is a local maximum of all the Burkholder functionals in (1.2).

From the point of view of the theory of nonlinear elasticity of Antman [3], Ball [9, 13], Ciarlet 24] and many others, for homogeneous materials the elastic deformations $f: \Omega \rightarrow \mathbb{R}^{n}$ are minimizers of a given energy integral

$$
\mathscr{E}[f]=\int_{\Omega} \mathbf{E}(D f) \mathrm{d} x<\infty
$$

where the so-called stored energy function $\mathbf{E}: \mathbb{R}^{n \times n} \rightarrow \mathbb{R}$ carries the mechanical properties of the elastic material in $\Omega$. By virtue of the principle of noninterpenetration of matter the minimizers ought to be injective. It is from these perspectives that our energy estimates, although limited to (quasiconformal) homeomorphisms, are certainly not short of applications. 
Among the strong consequences of the theorem, one obtains (with the same assumptions as in Theorem 1.3) that

$$
\frac{1}{|\Omega|} \int_{\Omega}|D f(z)|^{p} \mathrm{~d} z \leqslant \frac{2 K}{2 K-p(K-1)}, \quad \text { for } 2 \leqslant p<\frac{2 K}{K-1}
$$

with equality for piecewise power mappings, such as $f(z)=|z|^{1-1 / K} z$ in the unit disk; see Corollary 4.1 below. The $\mathscr{W}^{1, p}$-regularity of $K$-quasiconformal mappings, for $p<2 K /(K-1)$, was established by the first author in [4, as a corollary of his area distortion theorem. However, there the bounds for integrals such as in (1.5) were described in terms of unspecified constants depending on the distortion bound $K$. Here we have obtained the sharp explicit bound for the $\mathscr{L}^{p}$-integrals of the derivatives of $K$-quasiconformal mappings.

Similarly, for any $K$-quasiregular mapping $f \in \mathscr{W}_{\text {loc }}^{1,2}(\Omega)$, injective or not, we can improve the local $\mathscr{W}^{1, p}$-regularity to weighted integral bounds at the borderline exponent $p=2 K /(K-1)$,

$$
\left(\frac{1}{K(x)}-\frac{1}{K}\right)|D f(x)|^{\frac{2 K}{K-1}} \in \mathscr{L}_{\mathrm{loc}}^{1}(\Omega) .
$$

We refer to Section 3 for a more thorough discussion and to Section 5 for elaborate examples of extremal mappings.

We next describe shortly the ideas behind the proofs of our main results, which are given in Section 3 . In fact we will prove a slightly generalized form of Theorems 1.2 and 1.3, where we relax the identity boundary conditions to asymptotic normalization at infinity. This is done in Theorem 3.5 below, where we will interpolate between the natural end-point cases $p=2$ and $p=\infty$. The holomorphic interpolation method used is inspired by the variational principle of thermodynamical formalism and the underlying analytic dependence coming from holomorphic motions. The latter tools already figured prominently in the proof of the area distortion theorem [4] by the first author.

Here these are developed to a key ingredient of our argument, a new variant of the celebrated Riesz-Thorin interpolation theorem. We believe that the usefulness of this result may go beyond our interests here. In order to describe the result, let $\mathscr{M}(\Omega, \sigma)$ denote the class of complex-valued $\sigma$-measurable functions on a measure space $(\Omega, \sigma)$. The Lebesgue spaces $\mathscr{L}^{p}(\Omega, \sigma)$ are (quasi-)normed by

$$
\|\Phi\|_{p}=\left(\int_{\Omega}|\Phi(z)|^{p} \mathrm{~d} \sigma(z)\right)^{\frac{1}{p}}, \quad 0<p<\infty, \quad \text { and }\|\Phi\|_{\infty}=\operatorname{essip}_{z \in \Omega}|\Phi(z)| .
$$

Let $U \subset \mathbb{C}$ be a domain. We shall consider analytic families $f_{\lambda}$ of measurable functions in $\Omega$, i.e. jointly measurable functions $(x, \lambda) \mapsto f_{\lambda}(x)$ defined on $\Omega \times U$ such that for each fixed $x \in \Omega$ the map $\lambda \rightarrow f(x, \lambda)$ is analytic in $U$. The family is said to be nonvanishing if there exists a set $E \subset \Omega$ of $\sigma$-measure zero such that

$$
f(x, \lambda) \neq 0 \quad \text { for all } x \in \Omega \backslash E \text { and } \lambda \in U .
$$

We state our interpolation result first in the setting of the right half-plane, $U=$ $\mathbb{H}_{+}:=\{\lambda: \operatorname{Re} \lambda>0\}$, in order to facilitate comparison with the Riesz-Thorin theorem:

Lemma 1.4 (Interpolation Lemma). Let $0<p_{0}, p_{1} \leqslant \infty$ and let $\left\{\Phi_{\lambda}: \lambda \in\right.$ $\left.\mathbb{H}_{+}\right\} \subset \mathscr{M}(\Omega, \sigma)$ be an analytic and nonvanishing family, with complex parameter 
$\lambda$ in the right half-plane. Assume further that for some $a \geqslant 0$,

$$
M_{1}:=\left\|\Phi_{1}\right\|_{p_{1}}<\infty \text { and } M_{0}:=\sup _{\lambda \in \mathbb{H}_{+}} e^{-a \operatorname{Re} \lambda}\left\|\Phi_{\lambda}\right\|_{p_{0}}<\infty .
$$

Then, letting $M_{\theta}:=\left\|\Phi_{\theta}\right\|_{p_{\theta}}$ with $\quad \frac{1}{p_{\theta}}=(1-\theta) \cdot \frac{1}{p_{0}}+\theta \cdot \frac{1}{p_{1}}$, we have for every $0<\theta<1$,

$$
M_{\theta} \leqslant M_{0}^{1-\theta} \cdot M_{1}^{\theta}<\infty .
$$

Remark 1.5. Compared to Riesz-Thorin, our result needs the bound for the other end-point exponent only at one single point, when $\lambda=1$ ! However, without the nonvanishing condition the conclusion of the interpolation lemma breaks down drastically. A simple example (where $a=0$ ) is obtained by taking $p_{0}=1, p_{1}=\infty$, and considering the family $f(x, \lambda)=\left(\frac{1-\lambda}{1+\lambda}\right) g(x)$ for $\operatorname{Re} \lambda>0$ and $x \in \Omega$, for a function $g \in \mathscr{L}^{1}(\Omega, \sigma) \backslash\left(\bigcup_{p>1} \mathscr{L}^{p}(\Omega, \sigma)\right)$.

In applications one often has rotational symmetry, thus requiring a unit disk version of the interpolation. After a Möbius transform in the parameter plane the interpolation lemma runs as follows (observe that we have interchanged the roles of the indices $p_{0}$ and $p_{1}$ only for aesthetic reasons):

Lemma 1.6 (Interpolation Lemma for the disk). Let $0<p_{0}, p_{1} \leqslant \infty$ and $\left\{\Phi_{\lambda}\right.$ : $|\lambda|<1\} \subset \mathscr{M}(\Omega, \sigma)$ be an analytic and nonvanishing family with complex parameter $\lambda$ in the unit disc. Suppose

$$
M_{0}:=\left\|\Phi_{0}\right\|_{p_{0}}<\infty, \quad M_{1}:=\sup _{|\lambda|<1}\left\|\Phi_{\lambda}\right\|_{p_{1}}<\infty \quad \text { and } \quad M_{r}:=\sup _{|\lambda|=r}\left\|\Phi_{\lambda}\right\|_{p_{r}},
$$

where

Then, for every $0 \leqslant r<1$, we have

$$
\frac{1}{p_{r}}=\frac{1-r}{1+r} \cdot \frac{1}{p_{0}}+\frac{2 r}{1+r} \cdot \frac{1}{p_{1}} .
$$

$$
M_{r} \leqslant M_{0}^{\frac{1-r}{1+r}} \cdot M_{1}^{\frac{2 r}{1+r}}<\infty .
$$

As might be expected, passing to the limit in (1.3) as $p \rightarrow 2$ or as $p \rightarrow \infty$ will yield interesting sharp inequalities. The first mentioned limit leads to

Corollary 1.7. Given a bounded domain $\Omega \subset \mathbb{R}^{2}$ and a homeomorphism $f$ : $\Omega \stackrel{\text { onto }}{\longrightarrow} \Omega$ such that

we then have

$$
f(z)-z \in \mathscr{W}_{0}^{1,2}(\Omega)
$$

$$
\int_{\Omega}\left(1+\log |D f(z)|^{2}\right) J(z, f) \mathrm{d} z \leqslant \int_{\Omega}|D f(z)|^{2} \mathrm{~d} z
$$

Equality occurs for the identity map, as well as for a number of piecewise radial mappings discussed in Section 5 .

Reflecting back on Conjecture 1.1, note that the functional

$$
\mathscr{F}(A)=\left(1+\log |A|^{2}\right) \operatorname{det}(A)-|A|^{2}
$$

is rank-one concave. However, with growth stronger than quadratic it is not polyconcave 9], i.e. cannot be written as a concave function of the minors of $A$. According to Corollary 1.7 this functional is nevertheless quasiconcave with respect 
to homeomorphic perturbations of the identity. Going to the inverse maps yields a quasiconvexity result for the functional

$$
\mathscr{H}(A):=\frac{1}{2} \frac{|A|^{2}}{\operatorname{det} A}+\log (\operatorname{det} A)-\log |A|, \quad \operatorname{det} A>0 .
$$

This can be interpreted as a sharp integrability of $\log J(z, f)$ for planar maps of finite distortion; see Corollary 4.3 below.

It is of course classical [39, 28] that the nonlinear differential expression $J(z, f) \log |D f(z)|^{2}$ belongs to $\mathscr{L}_{\text {loc }}^{1}(\Omega)$ for every $f \in \mathscr{W}_{\text {loc }}^{1,2}(\Omega)$ whose Jacobian determinant $J(z, f)=\operatorname{det} D f(z)$ is nonnegative. The novelty in (1.10) lies in the best constant $C=1$ in the right hand side, and the proof of $\mathscr{L} \log \mathscr{L}$-integrability of the Jacobian is new.

In turn, the limit $p \rightarrow \infty$ yields the following sharp inequality.

Corollary 1.8. Denote by $\mathbf{S}$ the Beurling-Ahlfors operator (defined in (3.6)) and assume that $\mu$ is a measurable function with $|\mu(z)| \leqslant \chi_{\mathbb{D}}(z)$ for every $z \in \mathbb{C}$. Then

$$
\int_{\mathbb{D}}(1-|\mu(z)|) e^{|\mu(z)|}|\exp (\mathbf{S} \mu(z))| \mathrm{d} z \leqslant \pi .
$$

Equality occurs for an extensive class of piecewise radial mappings discussed in Section 5.2.

Prior to the above result, it was known that the area distortion results 4 yield the exponential integrability of $\operatorname{Re} \mathbf{S} \mu$ under the strict inequality $\|\mu\|_{\infty}<1$; see [5, p. $387]$.

The proofs of the corollaries are found in Section 4. Section 5 contains further observations on the Burkholder functionals. For example, their local maxima are discussed and new conjectures are posed also in the higher dimensional setup. We finally mention that an extension of Burkholder functionals to all real values of the exponent $p$ is given in Section 3 .

\section{Proof of the Interpolation Lemma}

Before embarking into the proof, let us remark that often analytic families of functions are defined by considering analytic functions having values in the Banach space $\mathscr{L}^{p}(\Omega, \sigma)$ for $p \geqslant 1$. In this case it is well known (e.g. [45, Thm. 3.31]) that one may define analyticity of the family by several equivalent conditions, e.g. by testing elements from the dual. This notion agrees with the definition given in the introduction; see [5, Lemma 5.7.1].

Proof of Lemma 1.4. We may, and do, assume that $M_{0}=1$ and that $a=0$; the case $a>0$ reduces to this case by simply considering the analytic family $e^{-a \lambda} \Phi_{\lambda}(x)$. Similarly by taking restrictions we may assume $\sigma(\Omega)<\infty$.

We first consider the case $0<p_{0}, p_{1}<\infty$, and establish the result in the situation where for a fixed $A \in(1, \infty)$ there is the uniform bound

$$
\frac{1}{A} \leqslant\left|\Phi_{\lambda}(x)\right| \leqslant A \quad \text { for all } \quad \lambda \in \mathbb{H}_{+} \text {and } x \in \Omega .
$$

This is to ensure that all of our integrals and computations below are meaningful. At the end of the proof we get rid of this extra assumption. 
Let $\theta \in(0,1)$ be given as in the statement of the lemma. First, we will find the support line to the convex function $\frac{1}{p} \mapsto \log \left\|\Phi_{\theta}\right\|_{p}$ at $\frac{1}{p_{\theta}}$. We are looking for a function $u_{p}(\theta)$ with the following properties:

$$
u_{p}(\theta)=\frac{1}{p} I+u_{\infty}(\theta) \leqslant \log \left\|\Phi_{\theta}\right\|_{p} \quad \text { and } \quad u_{p_{\theta}}(\theta)=\log \left\|\Phi_{\theta}\right\|_{p_{\theta}},
$$

where $I$ and $u_{\infty}(\theta)$ are independent of $p$. Using the concavity of the logarithm function we can write down these terms explicitly. Indeed, by concavity, for any probability density $\wp(x)$ on $\Omega$ and for any exponent $0<p<\infty$,

$$
\frac{1}{p} \int_{\Omega} \wp(x) \log \left(\frac{\left|\Phi_{\theta}(x)\right|^{p}}{\wp(x)}\right) \mathrm{d} \sigma \leqslant \log \left\|\Phi_{\theta}\right\|_{p},
$$

where equality holds for $p=p_{\theta}$ with the following choice of density:

$$
\wp(x):=\frac{\left|\Phi_{\theta}(x)\right|^{p_{\theta}}}{\int_{\Omega}\left|\Phi_{\theta}(y)\right|^{p_{\theta}} \mathrm{d} \sigma(y)}, \quad \int_{\Omega} \wp(x) \mathrm{d} \sigma(x)=1 .
$$

It is useful to note that because of our assumptions (2.1), the $\wp$ is uniformly bounded from above and below. With this in mind we find the coefficients in (2.2), by using the fixed density (2.3) and by writing

$$
I:=\int_{\Omega} \wp(x) \log \left(\frac{1}{\wp(x)}\right) \mathrm{d} \sigma \quad \text { and } \quad u_{\infty}(\theta):=\int_{\Omega} \wp(x) \log \left|\Phi_{\theta}(x)\right| \mathrm{d} \sigma .
$$

The key idea in this representation is that we may embed the line $u_{p}(\theta)$ in a harmonic family of lines parametrized by $\lambda \in \mathbb{H}_{+}$,

$$
u_{p}(\lambda):=\frac{1}{p} I+u_{\infty}(\lambda)=\frac{1}{p} \int_{\Omega} \wp(x) \log \left(\frac{\left|\Phi_{\lambda}(x)\right|^{p}}{\wp(x)}\right) \mathrm{d} \sigma .
$$

It is important to notice that we kept the slope $I$ fixed, and because of the nonvanishing assumption, the constant term $u_{\infty}(\lambda)$ becomes a harmonic function of $\lambda$. Again, in view of Jensen's inequality we have the envelope property, the analogue of (2.2) for all $\lambda \in \mathbb{H}_{+}$and $0<p \leqslant \infty$,

$$
u_{p}(\lambda) \leqslant \log \left\|\Phi_{\lambda}\right\|_{p} \quad \text { and } \quad u_{p_{\theta}}(\theta)=\log \left\|\Phi_{\theta}\right\|_{p_{\theta}} .
$$

By our assumptions, for $p=p_{0}$ we thus have $u_{p_{0}}(\lambda) \leqslant \log \left\|\Phi_{\lambda}\right\|_{p_{0}} \leqslant 0$ for all $\lambda \in \mathbb{H}_{+}$. Here Harnack's inequality for nonpositive harmonic functions in $\mathbb{H}_{+}$takes a particularly simple form when restricted to the interval $\theta \in(0,1)$ :

$$
u_{p_{0}}(\theta) \leqslant \theta u_{p_{0}}(1) \quad \text { for } \theta \in(0,1) .
$$

Finally combining the estimates (2.4) and (2.5) yields

$$
\begin{aligned}
\log \left\|\Phi_{\theta}\right\|_{p_{\theta}} & =u_{p_{\theta}}(\theta)=u_{p_{0}}(\theta)+\left(\frac{1}{p_{\theta}}-\frac{1}{p_{0}}\right) I \\
& \leqslant \theta u_{p_{0}}(1)+\theta\left(\frac{1}{p_{1}}-\frac{1}{p_{0}}\right) I \\
& =\theta u_{p_{1}}(1) \leqslant \theta \log M_{1}
\end{aligned}
$$

which is exactly what we aimed to prove.

The argument can easily be adapted to accommodate the cases when $p_{0}=\infty$ or $p_{1}=\infty$. We will instead use a limiting argument. First, normalize to $\sigma(\Omega)=1$; then $\|\cdot\|_{p}$ increases with $p$ and one has $\|f\|_{\infty}=\lim _{p \rightarrow \infty}\|f\|_{p}$. Hence, as (2.1) holds, 
we obtain the desired result by approximating the possibly infinite exponents by finite ones.

Let us finally dispense with the extra assumption (2.1). Since the removal of a null set from $\Omega$ is allowed, we may assume that the nonvanishing condition holds for every $x \in \Omega$; i.e., one may take $E=\emptyset$ in (1.7). Choose first a family $\varphi_{n}(\lambda)$ of Möbius transformations such that

$$
\varphi_{n}(1)=1, \quad \lim _{n \rightarrow \infty} \varphi_{n}(\lambda)=\lambda, \quad \lambda \in \mathbb{H}_{+}, \quad \text { and } \quad \overline{\varphi_{n}\left(\mathbb{H}_{+}\right)} \subset \mathbb{H}_{+}, \quad n \in \mathbb{N},
$$

and let for any positive integer $k$,

$$
\Omega_{n, k}:=\left\{x \in \Omega:\left|\Phi_{\lambda}(x)\right| \in[1 / k, k] \text { for all } \lambda \in \overline{\varphi_{n}\left(\mathbb{H}_{+}\right)}\right\} .
$$

The measurable sets $\Omega_{n, k}$ fill the space, $\bigcup_{k=1}^{\infty} \Omega_{n, k}=\Omega$. Moreover, for each fixed integer $k \geqslant 1$, the nonvanishing analytic family

$$
(x, \lambda) \mapsto \Phi_{\varphi_{n}(\lambda)}(x), \quad x \in \Omega_{n, k}, \quad \lambda \in \mathbb{H}_{+}
$$

satisfies the uniform bound (2.1), and thus we may interpolate it. Letting $k \rightarrow \infty$ gives $\left\|\Phi_{\varphi_{n}(\theta)}\right\|_{p_{\theta}} \leqslant M_{1}^{\theta}$, and the claim (1.8) follows by Fatou's lemma taking a second limit $n \rightarrow \infty$.

We remark that the Harnack inequality used above can be deduced from the standard Harnack inequality for negative harmonic functions in the unit disc, $u(w) \leqslant \frac{1-|w|}{1+|w|} u(0)$, by a change of variables $\lambda=(1-w) /(1+w)$. The very same change of variables allows one to deduce Lemma 1.6 for the values $\lambda \in(0,1)$ as a consequence of Lemma 1.4, and the rest follows from rotational symmetry.

\section{Proofs of the Main Theorems}

We start with some preliminaries. Our goal is to apply the Interpolation Lemma 1.6 in estimating the variational integrals such as (1.3), and therefore we look for analytic and nonvanishing families of gradients of mappings. In view of the Lambda Lemma [36] this takes us to the notion of quasiconformal mappings. By definition, in any dimension $n \geqslant 2$ these are homeomorphisms $f: \Omega \rightarrow \Omega^{\prime}$ in the Sobolev class $\mathscr{W}_{\text {loc }}^{1, n}(\Omega)$ for which the differential matrix $D f(x) \in \mathbb{R}^{n \times n}$ and its determinant are coupled in the distortion inequality,

$$
|D f(x)|^{n} \leqslant K(x) \operatorname{det} D f(x), \quad \text { where }|D f(x)|=\max _{|\xi|=1}|D f(x) \xi|,
$$

for some bounded function $K(x)$. The smallest $K(x) \geqslant 1$ for which (3.1) holds almost everywhere is referred to as the distortion function of the mapping $f$. We call $f K$-quasiconformal if $K(f):=\|K(x)\|_{\infty} \leqslant K$.

In dimension $n=2$ it is useful to employ complex notation by introducing the Cauchy-Riemann operators

$$
\partial f=f_{z}=\frac{\partial f}{\partial z}=\frac{1}{2}\left(\frac{\partial f}{\partial x}-i \frac{\partial f}{\partial y}\right) \quad \text { and } \quad \bar{\partial} f=f_{\bar{z}}=\frac{\partial f}{\partial \bar{z}}=\frac{1}{2}\left(\frac{\partial f}{\partial x}+i \frac{\partial f}{\partial y}\right) .
$$

Writing

$$
|D f(z)|=\left|f_{z}\right|+\left|f_{\bar{z}}\right| \quad \text { and } \quad \operatorname{det} D f(z)=J(z, f)=\left|f_{z}\right|^{2}-\left|f_{\bar{z}}\right|^{2},
$$

we see that for a planar Sobolev homeomorphism $f$ the $K$-quasiconformality simplifies to a linear equation

$$
f_{\bar{z}}=\mu(z) f_{z}
$$


called the Beltrami equation. Here the dilatation function $\mu$ is measurable and satisfies $\|\mu\|_{\infty}=: k=\frac{K-1}{K+1}<1$.

The Beltrami equation will then enable holomorphic deformations of the homeomorphism $f$ and of its gradient. Indeed, under a proper normalization, the solutions to (3.2) and their derivatives depend holomorphically on the coefficient $\mu$; see [5, p. 188].

For choosing the normalization, recall that Theorems 1.2 and 1.3 consider identity boundary values, and thus mappings that extend conformally outside $\Omega$. We therefore look for solutions to (3.2) defined in the entire plane $\mathbb{C}$, with the dilatation $\mu$ vanishing outside the domain $\Omega$. On the other hand, the identity boundary values cannot be retained under general holomorphic deformations; one needs to be content with the asymptotic normalization

$$
f(z)=z+b_{1} z^{-1}+b_{2} z^{-2}+\cdots, \text { for }|z| \rightarrow \infty .
$$

Global $\mathscr{W}_{\text {loc }}^{1,2}$-solutions to (3.2) with these asymptotics are called principal solutions. They exist and are unique for each coefficient $\mu$ supported in the bounded domain $\Omega$, and each of them is a homeomorphism. They can be found simply in the form of the Cauchy transform

$$
f(z)=z+\frac{1}{\pi} \int_{\mathbb{C}} \frac{\omega(\xi) \mathrm{d} \xi}{z-\xi}, \quad \text { where } \omega=f_{\bar{z}} \in \mathscr{L}^{2}(\mathbb{C}) .
$$

Substituting $\omega:=f_{\bar{z}}$ into (3.2) yields a singular integral equation for the unknown density function $\omega \in \mathscr{L}^{2}(\mathbb{C})$,

$$
\omega-\mu \mathbf{S} \omega=\mu \in \mathscr{L}^{2}(\mathbb{C}) .
$$

Here the Beurling Transform, a Calderón-Zygmund type singular integral,

$$
\mathbf{S} \omega=-\frac{1}{\pi} \int_{\mathbb{C}} \frac{\omega(\xi) \mathrm{d} \xi}{(z-\xi)^{2}}=f_{z}-1,
$$

is an isometry in $\mathscr{L}^{2}(\mathbb{C})$, whence $(3.5)$ can be solved by the Neumann series. We refer to the well-known monographs [1, 30] and [5] for the basic properties and further details on quasiconformal mappings.

Regularity results in $\mathscr{W}_{\text {loc }}^{1, p}$ originated in [18], [19], where $\mathbf{S}$ was considered on $\mathscr{L}^{p}$ for suitable exponents $p>2$. As a Calderón-Zygmund operator, $\mathbf{S}$ is bounded in $\mathscr{L}^{p}$, but determining here the operator norm is a much harder question. The as yet unsolved conjecture [31] of Iwaniec asserts that

Conjecture 3.1. For all $1<p<\infty$ the follow holds true:

$$
\|\mathbf{S}\|_{\mathscr{L}^{p}(\mathbb{C})}=p^{*}-1:=\left\{\begin{array}{cl}
p-1, & \text { if } 2 \leqslant p<\infty, \\
1 /(p-1), & \text { if } 1<p \leqslant 2 .
\end{array}\right.
$$

As mentioned in the introduction, the full quasiconcavity of the Burkholder functional $\mathbf{B}_{p}$ would, among its many potential consequences, imply also (3.7). This follows from another very useful inequality of Burkholder 23. Namely, with the positive constant $C_{p}=p\left(1-\frac{1}{p}\right)^{1-p}$ for $p \geqslant 2$, we have

$$
C_{p} \cdot\left(\left|f_{z}\right|^{p}-(p-1)^{p}\left|f_{\bar{z}}\right|^{p}\right) \leqslant\left(\left|f_{z}\right|-(p-1)\left|f_{\bar{z}}\right|\right) \cdot\left(\left|f_{z}\right|+\left|f_{\bar{z}}\right|\right)^{p-1} \equiv \mathbf{B}_{p}(D f) .
$$

Thus Burkholder's functional can be viewed as an effective rank-one concave majorant of the $p$-norm functional of the left hand side. It is because of this connection 
why Morrey's Problem becomes relevant to Conjecture 3.1. For precise statements and further information on related topics, we refer to [14, 16, 23, 47.

It is also appropriate to note that the origin of the Burkholder functional lies in Burkholder's groundbreaking work on sharp estimates for martingales [20]-23]. This work was later extended in various ways, including applications to computing optimal or almost optimal estimates for norms of singular integrals, e.g. of the Beurling-Ahlfors operator. Also the Bellman function techniques (see e.g. 41]) are closely related. We mention only [15, [17, 23], 25, 27, 44, 44, 44, 47] and refer to the recent survey [14] for a wealth of information and an extensive list of references.

We next recall some standard facts on smooth approximation of principal solutions of the Beltrami equation. Especially, we have a special interest in the Jacobian and whether it is strictly positive. Schauder's regularity theory of elliptic PDEs with Hölder continuous coefficients becomes useful. It applies to general quasilinear Beltrami systems (see Theorem 15.0.7 in [5])

$$
\frac{\partial f}{\partial \bar{z}}=\mu(z, f) \frac{\partial f}{\partial z}+\nu(z, f) \frac{\overline{\partial f}}{\partial z}, \quad|\mu(z, f)|+|\nu(z, f)| \leqslant k<1,
$$

where the coefficients $\mu, \nu \in \mathscr{C}^{\alpha}(\mathbb{C} \times \mathbb{C}), 0<\alpha<1$. Accordingly, every solution $f \in \mathscr{W}_{\text {loc }}^{1,2}(\mathbb{C} \times \mathbb{C})$ is $\mathscr{C}_{\text {loc }}^{1, \alpha}$-regular. Of course, if $f$ is quasiconformal, then the inverse map $h(w)=f^{-1}(w)$ is also $\mathscr{C}_{\text {loc }}^{1, \alpha}$-regular since it solves the similar system

$$
\frac{\partial h}{\partial \bar{w}}=-\nu(h, w) \frac{\partial h}{\partial w}-\mu(h, w) \frac{\overline{\partial h}}{\partial w} .
$$

Thus, we have

Lemma $3.2\left(\mathscr{C}^{1, \alpha}\right.$-regularity). The principal solution of the Beltrami equation (3.2) in which $\mu \in \mathscr{C}^{\alpha}(\mathbb{C}), 0<\alpha<1$, is a $\mathscr{C}^{1, \alpha}(\mathbb{C})$-diffeomorphism. In particular, $\left|f_{z}\right|^{2} \geqslant J(z, f)>0$, everywhere.

As might be expected, almost everywhere convergence of the Beltrami coefficients yields $\mathscr{W}_{\text {loc }}^{1,2}$-convergence of the principal solutions. The precise statement reads as follows:

Lemma 3.3 (Smooth approximation). Suppose the Beltrami coefficients $\mu_{\ell} \in$ $\mathscr{C}_{\circ}^{\infty}(\Omega)$ satisfy $\left|\mu_{\ell}(z)\right| \leqslant k<1$, for all $\ell=1,2, \ldots$, and converge almost everywhere to $\mu$. Then the associated principal solutions $f^{\ell}: \mathbb{C} \rightarrow \mathbb{C}$ are $\mathscr{C}^{\infty}$-smooth diffeomorphisms converging in $\mathscr{W}_{\mathrm{loc}}^{1,2}(\mathbb{C})$ to the principal solution of the limit equation $f_{\bar{z}}=\mu(z) f_{z}$.

Every measurable Beltrami coefficient satisfying $|\mu(z)| \leqslant k \chi_{\Omega}(z), \quad 0 \leqslant k<1$, can be approximated this way.

As the last of the preliminaries, in applying the Interpolation Lemma 1.6 we will need sharp $\mathscr{L}^{2}$-estimates of gradients, valid for all complex deformations of a given mapping. For the principal solutions in the unit disk $\mathbb{D}$, these result from the classical Area Theorem (see, e.g., 5, p. 41]).

Lemma 3.4 (Area inequality). The area of the image of the unit disk under a principal solution in $\mathbb{D}$ does not exceed $\pi$. It equals $\pi$ if and only if the solution is the identity map outside the disk. 
Let us recall a proof emphasizing the null-Lagrangian property of the Jacobian determinant. On the circle we have the equality $f(z)=g(z)$, where $g \in \mathscr{W}^{1,2}(\mathbb{D})$ is given by $g(z)=z+\sum_{n \geqslant 1} b_{n} \bar{z}^{n}$, for $|z| \leqslant 1$. This yields

$$
\int_{\mathbb{D}} J(z, f) \mathrm{d} z=\int_{\mathbb{D}} J(z, g) \mathrm{d} z=\int_{\mathbb{D}}\left(1-\left|g_{\bar{z}}(z)\right|^{2}\right) \mathrm{d} z \leqslant \pi .
$$

Equality occurs if and only if $g_{\bar{z}} \equiv 0$, meaning that all the coefficients $b_{n}$ vanish.

Having disposed of these lemmas, we can now proceed to the proof of the main integral estimate, where in the complex notation the Burkholder functional takes the form

$$
\mathscr{B}_{\Omega}^{p}[f]:=\int_{\Omega}\left(\left|f_{z}\right|-(p-1)\left|f_{\bar{z}}\right|\right) \cdot\left(\left|f_{z}\right|+\left|f_{\bar{z}}\right|\right)^{p-1} \mathrm{~d} z, \quad p \geqslant 2 .
$$

We will actually deduce (1.3) from a slightly more general result, where we relax the identity boundary values and allow principal mappings:

Theorem 3.5 (Sharp $\mathscr{L}^{p}$-inequality). Let $f: \mathbb{C} \rightarrow \mathbb{C}$ be the principal solution of a Beltrami equation,

$$
f_{\bar{z}}(z)=\mu(z) f_{z}(z), \quad|\mu(z)| \leqslant k \chi_{\mathbb{D}}(z), \quad 0 \leqslant k<1,
$$

in particular, conformal outside the unit disk $\mathbb{D}$.

Then, for all exponents $2 \leqslant p \leqslant 1+1 / k$, we have

$$
\int_{\mathbb{D}}\left(1-\frac{p|\mu(z)|}{1+|\mu(z)|}\right)\left(\left|f_{z}(z)\right|+\left|f_{\bar{z}}(z)\right|\right)^{p} \mathrm{~d} z \leqslant \pi .
$$

Equality occurs for some fairly general piecewise radial mappings discussed in Section 5 .

The above form of the main result gives a flexible and remarkably precise local description of the $\mathscr{L}^{p}$-properties of derivatives of a quasiconformal map, especially interesting in the borderline situation $p=1+1 / k$. Indeed, combined with the Stoilow factorization, the theorem gives for any $\mathscr{W}_{\text {loc }}^{1,2}(\Omega)$-solution to (3.9), injective or not, the estimate

$$
(k-|\mu(z)|)|D f(z)|^{1+1 / k} \in \mathscr{L}_{\text {loc }}^{1}(\Omega) .
$$

Thus for all $K$-quasiregular mappings we obtain optimal weighted higher integrability bounds at the borderline case $p=2 K /(K-1)$. For $p$ below the borderline, the $\mathscr{W}_{\text {loc }}^{1, p}$-regularity was established already in [4. The borderline integrability was previously covered [7] only in the very special case $|\mu|=k \cdot \chi_{E}$, for $E \subset \mathbb{D}$, in Theorem 3.5 .

The proof of Theorem 3.5 applies the Interpolation Lemma in conjunction with analytic families of quasiconformal maps. However, the choice of the specific analytic family for our situation is quite nontrivial, in order to enable sharp estimates. In a sense the speed of the change with respect to the analytic parameter must be localized in a delicate manner; see (3.14) below.

Proof of Theorem 3.5. Given a principal solution $f$ to (3.9), with $\mu(z) \equiv 0$ for $|z|>1$, we are to prove the integral bounds (3.10). There is no loss of generality in assuming that $\mu \in \mathscr{C}_{0}^{\infty}(\mathbb{D})$, for if not, we approximate $\mu$ with $\mathscr{C}_{0}^{\infty}$-smooth Beltrami coefficients, and, thanks to Fatou's lemma, there is no difficulty in passing 
to the limit in (3.10). On the other hand, this reduction could be avoided by using the argument of Remark 3.6 below.

With this assumption we fix an exponent $2 \leqslant p \leqslant 1+\|\mu\|_{\infty}^{-1}$ and look for holomorphic deformations of the given function $f$, via an analytic family of Beltrami equations together with their principal solutions,

$$
F_{\bar{z}}^{\lambda}=\mu_{\lambda}(z) F_{z}^{\lambda}, \quad \mu_{\lambda}(z)=\tau_{\lambda}(z) \cdot \frac{\mu(z)}{|\mu(z)|} .
$$

Here $\tau_{\lambda}(z)$ is an analytic function in $\lambda$ to be chosen later with $\left|\tau_{\lambda}(z)\right|<1$. We aim to explore Interpolation in the disk, Lemma 1.6. by applying it to a suitable nonvanishing analytic family constructed from the derivatives of $F^{\lambda}(z)$. Hence the question is the right choice of $\tau_{\lambda}$.

We want $F^{0}(z) \equiv z$, thus $\tau_{0}(z) \equiv 0$, while for some value $\lambda=\lambda_{\circ}$ we need to have $\tau_{\lambda_{\circ}}(z)=|\mu(z)|$, so that $f=F^{\lambda_{\circ}}$. Comparing the exponents in (3.10) and in Lemma 1.6] suggests that we choose

$$
p=1+\frac{1}{\lambda_{\circ}}, \quad p_{0}=\infty, \quad p_{1}=2 .
$$

These conditions will then be confronted with the need of weighted $\mathscr{L}^{2}$-bounds consistent with the inequality (3.10).

To make the long story short, we choose

$$
\mu_{\lambda}(z)=\tau_{\lambda}(z) \cdot \frac{\mu(z)}{|\mu(z)|}, \quad \text { where } \frac{\tau_{\lambda}(z)}{1+\tau_{\lambda}(z)}=p \cdot \frac{|\mu(z)|}{1+|\mu(z)|} \cdot \frac{\lambda}{1+\lambda},
$$

or more explicitly,

$$
\mu_{\lambda}(z)=\frac{p \lambda \mu(z)}{(1+\lambda)(1+|\mu(z)|)-p \lambda|\mu(z)|} .
$$

The complex parameter $\lambda$ runs over the unit disk, $|\lambda|<1$. One may visualize $\lambda \mapsto \tau_{\lambda}(z)$ as the conformal mapping from the unit disk onto the horocycle

$$
\left\{w \in \mathbb{D}: 2 \operatorname{Re}\left(\frac{w}{1+w}\right)<p \cdot \frac{|\mu(z)|}{1+|\mu(z)|}\right\}
$$

determined by the weight function in (3.10).

From (3.14) one readily sees that $\left|\mu_{\lambda}(z)\right| \leqslant|\lambda| \chi_{\mathbb{D}}(z)$; furthermore $\mu_{\lambda} \in \mathscr{C}^{\alpha}(\mathbb{C})$, $0<\alpha \leqslant 1$. Therefore the equation (3.12) admits a unique principal solution

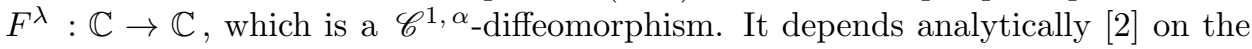
parameter $\lambda$, as seen by developing (3.5) in a Neumann series, and we have

$$
\left|F_{z}^{\lambda}\right|^{2} \geqslant\left|F_{z}^{\lambda}\right|^{2}-\left|F_{\bar{z}}^{\lambda}\right|^{2}=J\left(z, F^{\lambda}\right)>0, \text { everywhere in } \mathbb{C} .
$$

Moreover, $F^{0}(z)=z$ with $F^{\lambda_{\circ}}=f$, where $\lambda_{\circ}$ was defined by (3.13).

As the nonvanishing analytic family $\left\{\Phi_{\lambda}\right\}_{|\lambda|<1}$ we choose

$$
\Phi_{\lambda}(z)=F_{z}^{\lambda}(z)\left(1+\tau_{\lambda}(z)\right) .
$$

Explicitly,

$$
\Phi_{\lambda}(z)=\frac{(1+\lambda)(1+|\mu(z)|) F_{z}^{\lambda}(z)}{(1+\lambda)(1+|\mu(z)|)-p \lambda|\mu(z)|} \neq 0, \quad \text { for all } z \in \mathbb{D} .
$$

Furthermore, since $F^{\lambda_{\circ}} \equiv f, F_{z}^{\lambda_{\circ}} \equiv f_{z}$,

$$
\left|\Phi_{\lambda_{\diamond}}(z)\right|=(1+|\mu(z)|)\left|f_{z}\right|=\left|f_{z}\right|+\left|f_{\bar{z}}\right|=|D f| .
$$


We shall then apply the Interpolation Lemma 1.6 in the measure space $\mathscr{M}(\mathbb{D}, \sigma)$ over the unit disk, where

$$
\mathrm{d} \sigma(z)=\frac{1}{\pi}\left(1-\frac{p|\mu(z)|}{1+|\mu(z)|}\right) \mathrm{d} z .
$$

We start with the centerpoint $\lambda=0$, where the Beltrami equation reduces to the complex Cauchy-Riemann system $F_{\bar{z}} \equiv 0$ with principal solution the identity map. Hence $F_{z}^{0} \equiv 1, \Phi_{0}(z) \equiv 1$ and $M_{0}=\left\|\Phi_{0}\right\|_{\infty}=1$.

The estimate $M_{1}=\sup _{\lambda \in \mathbb{D}}\left\|\Phi_{\lambda}\right\|_{2} \leqslant 1$ requires just a bit more work. First, in view of Lemma 3.4

$$
\int_{\mathbb{D}} J\left(z, F^{\lambda}\right) \mathrm{d} z \leqslant \pi
$$

with equality if and only if $F^{\lambda}(z) \equiv z$ outside the unit disk. Here we find from (3.14) that

$$
\begin{aligned}
J\left(z, F^{\lambda}\right) & =\left|F_{z}^{\lambda}(z)\right|^{2}\left(1-\left|\mu_{\lambda}(z)\right|^{2}\right)=\left|\Phi_{\lambda}(z)\right|^{2}\left(1-2 \operatorname{Re} \frac{\tau_{\lambda}(z)}{1+\tau_{\lambda}(z)}\right) \\
& =\left|\Phi_{\lambda}(z)\right|^{2}\left(1-p \frac{|\mu(z)|}{1+|\mu(z)|} \operatorname{Re} \frac{2 \lambda}{1+\lambda}\right) \geqslant\left|\Phi_{\lambda}(z)\right|^{2}\left(1-p \frac{|\mu(z)|}{1+|\mu(z)|}\right) .
\end{aligned}
$$

Hence

and, therefore,

$$
\left|\Phi_{\lambda}(z)\right|^{2} \mathrm{~d} \sigma(z) \leqslant \frac{1}{\pi} J\left(z, F^{\lambda}\right) \mathrm{d} z
$$

$$
M_{1}=\sup _{|\lambda|<1} \int_{\mathbb{D}}\left|\Phi_{\lambda}(z)\right|^{2} \mathrm{~d} \sigma(z) \leqslant \sup _{|\lambda|<1} \frac{1}{\pi} \int_{\mathbb{D}} J\left(z, F^{\lambda}\right) \mathrm{d} z \leqslant 1 .
$$

We are now ready to interpolate. For every $0 \leqslant r<1$, in view of the Interpolation Lemma, we have

$$
M_{r}=\sup _{|\lambda|=r}\left\|\Phi_{\lambda}\right\|_{\frac{1+r}{r}} \leqslant M_{0}^{\frac{1-r}{1+r}} M_{1}^{\frac{2 r}{1+r}} \leqslant 1 .
$$

It remains to substitute $r=\frac{1}{p-1}=\lambda_{\circ}$. The desired inequality is now immediate,

$$
\int_{\mathbb{D}}\left(1-\frac{p|\mu(z)|}{1+|\mu(z)|}\right)|D f(z)|^{p} \mathrm{~d} z=\pi \int_{\mathbb{D}}\left|\Phi_{\lambda_{\circ}}(z)\right|^{\frac{1+r}{r}} \mathrm{~d} \sigma(z) \leqslant \pi .
$$

Proof of Theorems 1.2 and 1.3. To infer Theorem 1.3 we extend $f$ as the identity outside $\Omega$. Since $\Omega$ is bounded, $\int_{\Omega}|D f|^{2} \leqslant K \int_{\Omega} J(x, f)<\infty$ so that $f \in \mathscr{W}^{1,2}(\Omega)$. But then one easily verifies that the extended function $f$ defines an element of $\mathscr{W}_{\text {loc }}^{1,2}(\mathbb{C})$, and accordingly, a $K$-quasiconformal map of the entire plane.

Consider then a disk $D_{R} \supset \Omega$. By rescaling, if necessary, Inequality (3.10) applies to $D_{R}$ in place of the unit disk and with $\left|D_{R}\right|$ in place of $\pi$. This yields $\mathscr{B}_{D_{R}}^{p}[f] \leqslant \mathscr{B}_{D_{R}}^{p}[\mathrm{Id}]$. On the other hand, $\mathscr{B}_{D_{R} \backslash \Omega}^{p}[f]=\mathscr{B}_{D_{R} \backslash \Omega}^{p}[\mathrm{Id}]$, by trivial means. Hence Theorem 1.3 follows.

Theorem 1.2, in turn, is a direct consequence of Theorem 1.3. The pointwise condition $\mathbf{B}_{p}(D f) \geqslant 0$ for $f=z+h(z)$, together with the boundary condition $h \in$ $\mathscr{C}_{0}^{\infty}(\Omega)$, ensures that $f$ represents a (smooth) $K$-quasiconformal homeomorphism of $\Omega$ having identity boundary values. Here $p$ and $K$ are related by $p=2 K /(K-1)$ and we may apply Theorem 1.3 at the borderline exponent. 
Remark 3.6. Above we employed a standard approximation by smooth homeomorphisms in order to easily ensure that our analytic family of quasiconformal maps has nonvanishing Jacobian everywhere. It might be of interest to observe that actually all analytic families of $\partial_{z}$-derivatives of quasiconformal maps are nonvanishing, i.e. nonvanishing outside a common set of measure zero. This can be deduced via a standard reduction from the following result:

Lemma 3.7. Assume that the measurable dilatation $\mu_{\lambda}$ depends analytically on the parameter $\lambda \in U$, where $U \subset \mathbb{C}$ is a domain. Suppose also that $\left|\mu_{\lambda}\right| \leqslant a \chi_{\Omega}$ for some $0 \leqslant a<1$, for all $\lambda \in U$.

Let $f=f(\lambda, z)$ be the principal solution of the Beltrami equation $f_{\bar{z}}=\mu_{\lambda} f_{z}$. Then $\lambda \rightarrow f_{z}$ yields a nonvanishing analytic family.

Proof. By localization we may assume that $U=\mathbb{D}$. If the $\mu_{\lambda}$ are smooth functions of the $z$-variable, the nonvanishing property is due to Lemma 3.2. For a general nonsmooth $\mu_{\lambda}$ we introduce the mollification $\mu_{\lambda, j}:=\phi_{1 / j} * \mu_{\lambda}$, where $\phi_{\varepsilon}$ is a standard approximation of the identity. Then for any compact subset $K \subset \mathbb{D}$,

$$
\lim _{j \rightarrow \infty} \sup _{\lambda \in K}\left\|\mu_{\lambda, j}-\mu_{\lambda}\right\|_{\mathscr{L}^{p}(\Omega)}=0 \quad \text { for any } p>1 .
$$

Denoting by $f_{j}=f_{j}(\lambda, z)$ the principal solution corresponding to $\mu_{\lambda, j}$ and by choosing $p$ large enough, we may utilize [5, Lemma 5.3.1], to see that $\left\|\left(f_{j}\right)_{z}-f_{z}\right\|_{2} \rightarrow$ 0 , uniformly in $\lambda \in K$.

Next, developing the $\mathscr{L}^{2}(\Omega)$-valued analytic functions $f_{z}$ and $\left(f_{j}\right)_{z}$ as a power series in $\lambda$, we see from Cauchy's formula that the Taylor coefficients of $\left(f_{j}\right)_{z}$ converge in $\mathscr{L}^{2}(\Omega)$ to those of $f_{z}$. Thus, moving to a subsequence if needed, we have outside a set $E \subset \Omega$ of measure zero,

$$
\left(f_{j}\right)_{z}(\lambda, z) \rightarrow f_{z}(\lambda, z), \quad \text { local uniformly in } \lambda \in \mathbb{D} \text {. }
$$

Now, according to Hurwitz's theorem, the limit of a sequence of nonvanishing analytic functions converging locally uniformly is either everywhere nonzero, or identically zero. Choosing a parameter $\lambda_{0}$, the derivative $f_{z}\left(\lambda_{0}, \cdot\right)$ can vanish only on a set $E_{0}$ of measure zero [5, Corollary 3.7.6]. Thus $f_{z}(\lambda, z) \neq 0$ for every $\lambda \in \mathbb{D}$ and every $z \in \Omega \backslash\left(E \cup E_{0}\right)$.

The notions of quasiconvexity and rank-one convexity extend to functions defined on an open subset $\mathscr{O} \subset \mathbb{R}^{2 \times 2}$. Rank-one convexity now demands that for any $A \in \mathscr{O}$ and rank-one matrix $X \in \mathbb{R}^{2 \times 2}$ the map $t \mapsto E(A+t X)$ is convex in a neighbourhood of zero. The condition for being rank-one concave or null Lagrangian is modified analogously. Similarly, in the definition of quasiconvexity (1.1) one simply restricts to linear maps $A$ and perturbations $f \in A+\mathscr{C}_{0}^{\infty}(\Omega)$ such that $D f(z) \in \mathscr{O}$ for all $z \in \Omega$.

With the above generalized definitions in mind we next explore a dual formulation to Theorem 1.3. i.e., we will pass via the inverse map from expansion estimates to compression estimates. In doing so, we shall restrict to the space $\mathscr{O}=\mathbb{R}_{+}^{2 \times 2}$ consisting of matrices with positive Jacobian determinant. The inverse functional takes the form

$$
\hat{\mathbf{E}}(A):=\mathbf{E}\left(A^{-1}\right) \cdot \operatorname{det} A, \quad \operatorname{det} A>0,
$$

and it preserves rank-one convexity, quasiconvexity as well as polyconvexity [10, p. 211]. One should also note that for $\phi \in \mathscr{C}_{0}^{\infty}(\Omega)$ the condition $\operatorname{Df}(z)=A+$ $D \phi(z) \in \mathbb{R}_{+}^{2 \times 2}$ for all $z \in \Omega$ automatically implies that $f$ is a diffeomorphism 
$f: \Omega \rightarrow A(\Omega)$. This enables one to switch to an inverse map if needed. Our main observation here is that the procedure of taking inverse functionals naturally leads to a full one-parameter family of Burkholder functionals.

Let us recall the standard case (1.2) where one now includes all exponents $p \geqslant 1$,

$$
\mathbf{B}_{p}(A)=\left(\frac{p}{2} \operatorname{det} A+\left(1-\frac{p}{2}\right)|A|^{2}\right) \cdot|A|^{p-2}, \quad p \geqslant 1 .
$$

We set for $p \leqslant 1$,

$$
\mathbf{B}_{p}(A):=\left(\frac{p}{2}|A|^{2}+\left(1-\frac{p}{2}\right) \operatorname{det} A\right) \cdot|A|^{-p} \cdot(\operatorname{det} A)^{p-1}, \quad \operatorname{det} A>0 .
$$

Then the functionals $\mathbf{B}_{p}$ are homogeneous of degree $p$, depend continuously on $p$, and we have for the inverse transformation, $\hat{\mathbf{B}}_{p}=\mathbf{B}_{q}$, where $p+q=2$. These facts justify the definitions. Note that, in particular, we recover the null-Lagrangian cases $p=0$ and $p=2$ as smooth phase transition points between the rank-one convex and rank-one concave regimes:

Proposition 3.8. In the space of matrices $A \in \mathbb{R}_{+}^{2 \times 2}$,

$$
A \mapsto \boldsymbol{B}_{p}(A) \text { is } \begin{cases}\text { rank-one convex } & \text { if } 0 \leqslant p \leqslant 2, \\ \text { null-Lagrangian } & \text { if } p=0 \text { or } p=2, \\ \text { rank-one concave } & \text { if } p \leqslant 0 \text { or } p \geqslant 2 .\end{cases}
$$

The standard case of $p \geqslant 1$ above goes back to Burkholder [23] and the rest follows from applying the inverse transformation. In the complex notation we have, for any $p \in \mathbb{R}$,

$$
\mathscr{B}_{\Omega}^{p}[f]=\int_{\Omega} \mathbf{B}_{p}(D f)=\int_{\Omega}\left(\left|f_{z}\right| \mp(p-1)\left|f_{\bar{z}}\right|\right) \cdot\left(\left|f_{z}\right| \pm\left|f_{\bar{z}}\right|\right)^{p-1} \mathrm{~d} z,
$$

where \pm stands for the sign of $(p-1)$.

Another application of the inverse transformation, this time to Theorem 1.3. leads to

Theorem 3.9. Let $f: \Omega \longrightarrow \Omega$ be a $K$-quasiconformal map of a bounded open set $\Omega \subset \mathbb{C}$ onto itself, extending continuously up to the boundary, where it coincides with the identity map $\operatorname{Id}(z) \equiv z$. Then

$$
\mathscr{B}_{\Omega}^{p}[f] \leqslant \mathscr{B}_{\Omega}^{p}[\mathrm{Id}] \text { for all }-\frac{2}{K-1} \leqslant p \leqslant 0 .
$$

Further, the equality occurs for a class of (compressing) piecewise radial mappings discussed in Section 5 .

\section{Sharp $\mathscr{L} \log \mathscr{L}, \mathscr{L}^{p}$ And exponential integrability}

The sharp integral inequalities provided by Theorems 3.5 and 1.3 give us a number of interesting consequences. We start with the following optimal form of the Sobolev regularity of $K$-quasiconformal mappings.

Corollary 4.1. Suppose $\Omega \subset \mathbb{C}$ is any bounded domain and $f: \Omega \rightarrow \Omega$ is a $K$-quasiconformal mapping, continuous up to $\partial \Omega$, with $f(z)=z$ for $z \in \partial \Omega$. Then

$$
\frac{1}{|\Omega|} \int_{\Omega}|D f(z)|^{p} \mathrm{~d} z \leqslant \frac{2 K}{2 K-p(K-1)}, \quad \text { for } 2 \leqslant p<\frac{2 K}{K-1} .
$$

The estimate holds as an equality for $f(z)=z|z|^{1 / K-1}, z \in \mathbb{D}$, as well as for a family of more complicated maps described in Section 5.2 . 
Proof. Inequality (4.1) is a straightforward consequence of Theorem 1.3, since for $p<2 K /(K-1)$ we have pointwise $\mathbf{B}_{p}(D f(x)) \geqslant|D f(x)|^{p} \frac{2 K-p(K-1)}{2 K}$.

We next introduce yet another rank-one concave variational integral, simply by differentiating $\mathscr{B}_{\Omega}^{p}[f]$ at $p=2$,

$$
\begin{aligned}
\mathscr{F}_{\Omega}[f] & :=\lim _{p \searrow 2} \frac{\mathscr{B}_{\Omega}^{p}[f]-\mathscr{B}_{\Omega}^{2}[f]}{p-2} \\
& =\frac{1}{2} \int_{\Omega}\left[\left(1+\log |D f(z)|^{2}\right) J(z, f)-|D f(z)|^{2}\right] \mathrm{d} z .
\end{aligned}
$$

The nonlinear differential expression $J(z, f) \log |D f(z)|^{2}$, for mappings with nonnegative Jacobian, is well known to be locally integrable. See 28 for the following qualitative local estimate on concentric balls $B \subset 2 B \subset \Omega$ :

$$
f_{B} J(z, f) \log \left(e+\frac{|D f(z)|^{2}}{f_{B}|D f|^{2}}\right) \mathrm{d} z \leqslant C f_{2 B}|D f(z)|^{2} \mathrm{~d} z
$$

see also Theorem 8.6.1 in [34. However, for global estimates one must impose suitable boundary conditions on $f$. For example, global $\mathscr{L} \log \mathscr{L}(\Omega)$ estimates follow from (4.3) if $f$ extends beyond the boundary of $\Omega$ with finite Dirichlet energy and nonnegative Jacobian determinant. This is the case, in particular, when $f(z)-z \in \mathscr{W}_{0}^{1,2}(\Omega)$.

Let us denote the class of homeomorphisms $f \in \mathscr{W}_{\text {loc }}^{1,2}(\mathbb{C})$ which coincide with the identity map outside a compact set by $\mathscr{W}_{\text {id }}^{1,2}(\mathbb{C})$. It is useful to observe (see e.g. [5. Thm. 20.1.6]) that such a map is automatically uniformly continuous. Further, the $\mathscr{C}^{\infty}$-smooth diffeomorphisms in $\mathscr{W}_{\text {id }}^{1,2}(\mathbb{C})$ are dense. Precisely, one has

Lemma 4.2 (Approximation Lemma, [33, Thm. 1]). Given any homeomorphism $f \in \mathscr{W}_{\mathrm{id}}^{1,2}(\mathbb{C})$, one can find $\mathscr{C}^{\infty}$-smooth diffeomorphisms $f^{\ell} \in \mathscr{W}_{\mathrm{id}}^{1,2}(\mathbb{C}), \ell \geqslant 1$, such that

$$
\left\|f^{\ell}-f\right\|_{\infty}+\left\|D\left(f^{\ell}-f\right)\right\|_{\mathscr{L}^{2}(\mathbb{C})} \rightarrow 0, \quad \text { as } \ell \rightarrow \infty .
$$

Passing to a subsequence if necessary, we may ensure that $D f^{\ell} \longrightarrow D f$ almost everywhere.

With estimates for the Burkholder integrals we now arrive at sharp global $\mathscr{L} \log \mathscr{L}(\Omega)$ bounds.

Proof of Corollary [1.7, Upon the extension as identity outside $\Omega, f \in \mathscr{W}_{\text {id }}^{1,2}(\mathbb{C})$. We use the sequence $\left\{f^{\ell}\right\}$ in the Approximation Lemma 4.2, and view each $f^{\ell}$ as a principal solution to its own Beltrami equation

$$
f_{\bar{z}}^{\ell}=\mu_{\ell}(z) f_{z}^{\ell}, \quad\left|\mu_{\ell}(z)\right| \leqslant k_{\ell}<1, \quad \mu_{\ell}(z)=0, \text { for }|z| \geqslant R
$$

where $R$ is chosen, and temporarily fixed, large enough so that $\Omega \subset D_{R}=\{z$ : $|z|<R\}$. It is legitimate to apply Theorem 1.3 for each of the maps $f^{\ell}: D_{R} \rightarrow$ $D_{R}$,

$$
\mathscr{B}_{D_{R}}^{p}\left[f^{\ell}\right] \leqslant\left|D_{R}\right|=\mathscr{B}_{D_{R}}^{2}\left[f^{\ell}\right], \quad \text { whenever } 2 \leqslant p \leqslant 1+k_{\ell}^{-1} .
$$

Letting $p \searrow 2$ we obtain

$$
\int_{D_{R}}\left(1+\log \left|D f^{\ell}(z)\right|^{2}\right) J\left(z, f^{\ell}\right) \mathrm{d} z \leqslant \int_{D_{R}}\left|D f^{\ell}(z)\right|^{2} \mathrm{~d} z
$$


Convergence theorems in the theory of integrals let us pass to the limit when $\ell \rightarrow$ $\infty$, as follows:

$$
\begin{array}{rl}
\int_{D_{R}} & J(z, f)\left[1+\log |D f(z)|^{2}\right] \mathrm{d} z \\
= & \int_{D_{R}} J(z, f)\left[1+\log \left(1+|D f|^{2}\right)\right] \mathrm{d} z-\int_{D_{R}} J(z, f)\left[\log \left(1+|D f|^{-2}\right)\right] \mathrm{d} z \\
\leqslant & \liminf _{\ell \rightarrow \infty} \int_{D_{R}} J\left(z, f^{\ell}\right)\left[1+\log \left(1+\left|D f^{\ell}\right|^{2}\right)\right] \mathrm{d} z \\
& \quad-\lim _{\ell \rightarrow \infty} \int_{D_{R}} J\left(z, f^{\ell}\right) \log \left(1+\left|D f^{\ell}\right|^{-2}\right) \mathrm{d} z
\end{array}
$$

Here the (lim inf)-term is justified by Fatou's theorem while the (lim)-term by the Lebesgue dominated convergence, where we observe that the integrand is dominated pointwise by $J\left(z, f^{\ell}\right)\left|D f^{\ell}\right|^{-2} \leqslant 1$. The lines of computation continue as follows:

$$
\begin{aligned}
& =\liminf _{\ell \rightarrow \infty} \int_{D_{R}} J\left(z, f^{\ell}\right)\left[1+\log \left|D f^{\ell}\right|^{2}\right] \mathrm{d} z \\
& \leqslant \liminf _{\ell \rightarrow \infty} \int_{D_{R}}\left|D f^{\ell}\right|^{2} \mathrm{~d} z=\int_{D_{R}}|D f(z)|^{2} \mathrm{~d} z .
\end{aligned}
$$

Finally, we observe that

$$
\int_{D_{R} \backslash \Omega} J(z, f)\left[1+\log |D f(z)|^{2}\right] \mathrm{d} z=\int_{D_{R} \backslash \Omega}|D f(z)|^{2} \mathrm{~d} z,
$$

which combined with the previous estimate yields (1.10), as desired.

The above energy functional can be further cultivated by applying the inverse map, as described at the end of Section 3. A search of minimal regularity in the corresponding integral estimates leads us to mappings of integrable distortion.

Corollary 4.3. Let $\Omega \subset \mathbb{R}^{2}$ be a bounded domain, and suppose $h \in \mathscr{W}_{\text {loc }}^{1,1}(\Omega)$ is a homeomorphism $h: \bar{\Omega} \stackrel{\text { onto }}{\rightarrow} \bar{\Omega}$ such that $h(z)=z$ for $z \in \partial \Omega$. Assume $h$ satisfies the distortion inequality

$$
|D h(z)|^{2} \leqslant K(z) J(z, h), \quad \text { a.e. in } \Omega,
$$

where $1 \leqslant K(z)<\infty$ almost everywhere in $\Omega$. The smallest such function, denoted by $K(z, h)$, is assumed to be integrable. Then

$$
2 \int_{\Omega}[\log |D h|-\log J(z, h)] \mathrm{d} z \leqslant \int_{\Omega}[K(z, h)-J(z, h)] \mathrm{d} z .
$$

In particular, $\log J(z, h)$ is integrable. Again there is a wealth of functions, to be described in Section 5, satisfying (4.5) as an identity.

Note that in fact

$$
K(z, h)= \begin{cases}\frac{|D h(z)|^{2}}{J(z, h)}, & \text { if } J(z, h)>0 \\ 1, & \text { otherwise. }\end{cases}
$$

Thus according to the corollary, the functional $\mathscr{H}(A)$ in (1.11) is quasiconvex at $A=\mathrm{Id}$, in its entire natural domain of definition $\mathscr{O}=\mathbb{R}_{+}^{2 \times 2}$. 
Proof of Corollary 4.3. It was observed in [6] that if $h: \Omega \rightarrow h(\Omega)$ is a homeomorphism of Sobolev class $\mathscr{W}_{\text {loc }}^{1,2}(\Omega)$ with integrable distortion $K(z, h) \in \mathscr{L}^{1}(\Omega)$, then its inverse map $f=h^{-1}: h(\Omega) \stackrel{\text { onto }}{\rightarrow} \Omega$ belongs to $\mathscr{W}^{1,2}(h(\Omega))$ and one has

$$
\int_{h(\Omega)}|D f|^{2} \mathrm{~d} z=\int_{\Omega} K(z, h) \mathrm{d} z .
$$

Enhancement of this identity for mappings $h \in \mathscr{W}_{\text {loc }}^{1,1}(\Omega)$ is given in [29].

In our situation $f(z)-z=h^{-1}(z)-z$ is continuous in $\bar{\Omega}$, vanishes on $\partial \Omega$, and belongs to $\mathscr{W}^{1,2}(\Omega)$. It is well known that this implies $f(z) \in z+\mathscr{W}_{0}^{1,2}(\Omega)$. Thus it is legitimate to apply Corollary 1.7 to deduce

$$
\int_{\Omega}\left[1+2 \log \left(\frac{|D h|}{J(z, h)}\right)\right] \mathrm{d} z \leqslant \int_{\Omega} K(z, h) \mathrm{d} z .
$$

Here we used the identity $\left|A^{-1}\right|=|A| / \operatorname{det}(A)$ and a change of variable, made legitimate by the fact that $f$ is a homeomorphism of Sobolev class $\mathscr{W}^{1,2}(\Omega)$. The claim (4.5) obviously follows from (4.7), and Hadamard's inequality yields the logarithmic integrability,

$$
-\int_{\Omega} \log J(z, h) \mathrm{d} z \leqslant \int_{\Omega}[K(z, h)-1] \mathrm{d} z,
$$

with equality here for the identity mapping.

In particular, the Jacobian of a map with integrable distortion cannot approach zero too rapidly. This result is in fact known [35; the novelty in (4.5) is the sharpness.

We next turn to the exponential integrability results, which will follow from Theorem 3.5 at the limit $p \rightarrow \infty$.

Proof of Corollary 1.8, Let us assume we are given a function $\mu$, supported in $\mathbb{D}$ with $|\mu(z)| \leqslant 1$ for all $z \in \mathbb{D}$. We then consider the principal solution $f$ of the Beltrami equation $f_{\bar{z}}=\varepsilon \mu f_{z}$ and apply Theorem 3.5 with $k=\varepsilon$ and $p=1+1 / \varepsilon$ to obtain

$$
\int_{\mathbb{D}}\left(\frac{1-|\mu(z)|}{1+\varepsilon|\mu(z)|}\right)|D f(z)|^{1+1 / \varepsilon} \mathrm{d} z \leqslant \pi .
$$

By applying the Cauchy-Schwarz inequality and the $\mathscr{L}^{2}$-isometric property of $\mathbf{S}$, we see that for almost every $z \in \mathbb{D}$, developing (3.5) (3.6) to a Neumann series represents $f_{z}$ as a power series in $\varepsilon$, with convergence radius $\geqslant 1$. Hence

$$
f_{z}=1+\varepsilon \mathbf{S} \mu+O\left(\varepsilon^{2}\right) \quad \text { for a.e. } z \in \mathbb{D} .
$$

We may use this to compute pointwise

$$
\begin{aligned}
(1+1 / \varepsilon) \log |D f| & =(1+1 / \varepsilon)\left(\log (1+\varepsilon|\mu|)+\log \left|f_{z}\right|\right) \\
& =|\mu|+\operatorname{Re} \mathbf{S} \mu+O(\varepsilon) .
\end{aligned}
$$

Hence $|D f|^{1+1 / \varepsilon}=\exp (|\mu|+\operatorname{Re} \mathbf{S} \mu)+O(\varepsilon)$ and the desired result follows at the limit $\varepsilon \rightarrow 0$ by an application of Fatou's lemma on (4.8). 


\section{Piecewise RADial MAPpingS}

5.1. Examples of optimality in Theorems 1.2, 1.3 and 3.5. Our exposition here is slightly condensed since the basic principle behind these examples can be found already in the paper [8] of A. Baernstein and S. Montgomery-Smith, or in the paper 32 of the second author. Let us start by describing the building block of the maps that yields equality in our main result. For any $0 \leqslant r<R$ consider the radial map

$$
g(z)=\rho(|z|) \frac{z}{|z|}
$$

defined in the disc $\{|z| \leqslant R\}$. We assume that $\rho:[0, R] \rightarrow[0, R]$ is absolutely continuous and strictly increasing with $\rho(0)=0$, and that $\rho$ is linear on $[0, r]$. We first restrict ourselves to the situation $p \geqslant 1$, and then we need the following expanding assumption:

$$
\frac{\rho(t)}{t} \geqslant \dot{\rho}(t) \geqslant 0, \quad t \in(r, R)
$$

together with the normalization $\rho(R)=R$. Hence on the boundary the map $g$ coincides with the identity map, and if needed we may extend $g$ to the exterior $\{|z| \geqslant R\}$ by setting $g(z)=z$ for these values.

The differential of $g$ exhibits the following rank-one connections:

$$
D g(z)=\frac{\rho(|z|)}{|z|} \operatorname{Id}+\left(\dot{\rho}(|z|)-\frac{\rho(|z|)}{|z|}\right) \frac{z \otimes z}{|z|^{2}} .
$$

It is known, see [12, Proposition 3.4], that concavity along the indicated rankone lines already secures the quasiconcavity condition for the radial map $g$. In our situation the assumption (5.2) indeed ensures that the Burkholder integrals become linear on the rank-one segments displayed in (5.3), which implies

$$
\mathscr{B}_{B(0, R)}^{p}[g]=\mathscr{B}_{B(0, R)}^{p}[\mathrm{Id}] .
$$

Actually, a direct computation (see $\left[8,32\right.$ for details) using the formulas $g_{z}(z)=$ $\frac{1}{2}(\dot{\rho}(|z|)+\rho(|z|) /|z|)$ and $g_{\bar{z}}(z)=\frac{1}{2}(\dot{\rho}(|z|)-\rho(|z|) /|z|) z / \bar{z}$ yields

$$
\begin{aligned}
\mathscr{B}_{B(0, R)}^{p}[g] & =\pi \int_{0}^{R}\left(\frac{[\rho(t)]^{p}}{t^{p-2}}\right)^{\prime} \mathrm{d} t=\pi \frac{[\rho(R)]^{p}}{R^{p-2}}-\lim _{t \rightarrow 0^{+}} \pi \frac{[\rho(t)]^{p}}{t^{p-2}} \\
& =\pi R^{2}=|B(0, R)|=\mathscr{B}_{B(0, R)}^{p}[\mathrm{Id}] .
\end{aligned}
$$

The above computation indicates that if $r=0$, we must in addition require

$$
\rho(t)=o\left(t^{1-\frac{2}{p}}\right) \quad \text { as } t \rightarrow 0 .
$$

Assume then that $f_{0}(z)=a z+b$ is a (complex) linear map defined in a bounded domain $\Omega \subset \mathbb{C}$. Given $0 \leqslant r<R$ and a ball $B\left(z_{0}, R\right) \subset \Omega$ together with the increasing homeomorphism $\rho:[0, R] \rightarrow[0, R]$ and the radial map $g$ as discussed above, we may modify $f_{0}$ in $B\left(z_{0}, R\right)$ by defining

$$
f_{1}(z)= \begin{cases}f_{0}(z) & \text { if } z \notin \overline{B\left(z_{0}, R\right)}, \\ a g\left(z-z_{0}\right)+\left(a z_{0}+b\right) & \text { if } z \in B\left(z_{0}, R\right) .\end{cases}
$$

By scaling, (5.4) shows that we have $\mathscr{B}_{B\left(z_{0}, R\right)}^{p}\left[f_{1}\right]=\mathscr{B}_{B\left(z_{0}, R\right)}^{p}\left[f_{0}\right]$, and, consequently,

$$
\mathscr{B}_{\Omega}^{p}\left[f_{1}\right]=\mathscr{B}_{\Omega}^{p}\left[f_{0}\right]
$$




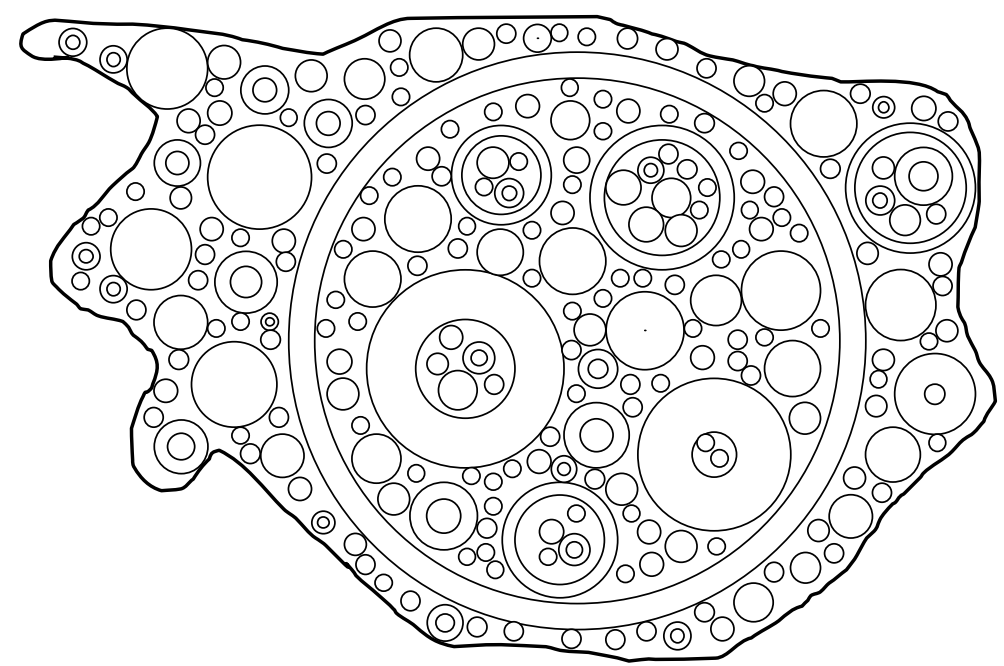

Figure 1. Annular Packing

In the next step we may deform $f_{1}$ in a disc that is contained in either one of the sets $\Omega \backslash B\left(z_{0}, R\right)$ or $B\left(z_{0}, r\right)$, where $f_{1}$ is linear. Inductively one obtains $f_{n}$ from $f_{n-1}$ by deforming $f_{n-1}$ accordingly in the domains of linearity. By induction, we see that all such mappings have the same energy, which is equal to the energy of their linear boundary data $a z+b$ :

$$
\mathscr{B}_{\Omega}^{p}\left[f_{n}\right]=\mathscr{B}_{\Omega}^{p}[a z+b]=a^{p}|\Omega| .
$$

This iteration process may, but need not, continue indefinitely so as to arrive at, e.g., a Cantor-type configuration of annuli and a homeomorphism $f_{\infty}: \Omega \stackrel{\text { onto }}{\longrightarrow}$ $\Omega^{*}:=a \Omega+b$. Without going to the formal definition, we loosely refer to reasonable (e.g. converging in $\mathscr{W}^{1,1}$ ) such limits $f=\lim _{n \rightarrow \infty} f_{n}$ as piecewise radial mappings; see Figure 1.

Definition 5.1. Let $p \geqslant 1$ and let $\Omega \subset \mathbb{C}$ be any nonempty bounded domain. The class $\mathscr{A}^{p}(\Omega)$ consists of piecewise radial mappings $f_{\infty}: \Omega \stackrel{\text { onto }}{\longrightarrow} \Omega$ whose construction starts with $f_{0}(z) \equiv z$, the convergence $f_{\infty}=\lim _{n \rightarrow \infty} f_{n}$ takes place in $\mathscr{W}^{1, p}(\Omega)$ and the condition (5.2) is in force.

The following observation is a direct corollary of (5.7).

Proposition 5.2. For any $p \geqslant 1$ and $f \in \mathscr{A}^{p}(\Omega)$ we have

$$
\mathscr{B}_{\Omega}^{p}[f]=\mathscr{B}_{\Omega}^{p}[\mathrm{Id}]=|\Omega| .
$$

In interpreting this conclusion one may say that $\mathscr{B}_{\Omega}^{p}$ is a null Lagrangian when restricted to $\mathscr{A}^{p}(\Omega)$.

We get a plethora of fairly complicated maps that produce equality in our main theorems. One just needs to consider any $f \in \mathscr{A}^{p}$ that satisfies the additional condition $\mathbf{B}_{p}(D f(x)) \geqslant 0$; i.e., in the construction one applies maps $\rho$ that satisfy

$$
\frac{\rho(t)}{t} \geqslant \dot{\rho}(t) \geqslant\left(1-\frac{2}{p}\right) \frac{\rho(t)}{t} .
$$


In the case of Theorem 1.2, in order to satisfy the smoothness assumption one of course has to pick the functions $\rho$ in the construction so that the (possibly limiting) map belongs to $I d+\mathscr{C}_{\circ}^{\infty}(\Omega)$.

In order to incorporate the range $p \leqslant 1$, one introduces the compressing assumption, the opposite of (5.2):

$$
\frac{\rho(t)}{t} \leqslant \dot{\rho}(t), \quad t \in(r, R)
$$

together with the condition $\lim _{t \rightarrow 0^{+}} \rho(t) / t^{1-2 / p}=\infty$ in case $r=0$ and $p<0$. The previous construction yields, after finitely many iterations, nontrivial examples of maps that satisfy (5.8) in case $p<1$. Also limiting maps can be considered, but then one has to take care of the Burkholder functional to remain well defined.

In view of Conjecture 1.1 the maps in $\mathscr{A}^{p}(\Omega)$ are potential global extremals for $\mathscr{B}_{\Omega}^{p}$. Indeed, it can be shown that they are critical points of the associated EulerLagrange equations. Furthermore, this property to a large extent characterizes Burkholder functionals: these functionals are the only (up to a scalar multiple) isotropic and homogeneous variational integrals with the $\mathscr{A}^{p}(\Omega)$ as their critical points. This topic will be discussed in a companion paper.

Here, we are content with pointing out the following result, where we employ the customary notation $\mathscr{C}_{\mathrm{id}}^{1}(\Omega)=\mathrm{id}+\mathscr{C}_{\mathrm{o}}^{1}(\Omega)$, where $\mathscr{C}_{\mathrm{o}}^{1}(\Omega)$ stands for the space of functions that are $\mathscr{C}^{1}$-smooth up to the boundary of $\Omega$ and vanishing on $\partial \Omega$.

Corollary 5.3. The Burkholder functional $\mathscr{B}_{\Omega}^{p}: \mathscr{C}_{\text {id }}^{1}(\Omega) \rightarrow \mathbb{R}, p>2$, attains its local maximum at every $\mathscr{C}^{1}$-smooth piecewise radial map in $\mathscr{A}^{p}(\Omega)$ for which the condition (5.9) is further reinforced to:

$$
\frac{\rho(t)}{t} \geqslant \dot{\rho}(t) \geqslant \frac{1}{K} \frac{\rho(t)}{t}, \quad K<\frac{p}{p-2} .
$$

Proof. The lower bound (5.11) can be used to verify that $f$ is $K$-quasiconformal with $K<p /(p-2)$. Namely, as $f \in \mathscr{C}_{\text {id }}^{1}(\Omega)$, one checks that $f$ is necessarily conformal at points corresponding to $t=0$ and the derivative is nonvanishing. Hence the strict inequality for $K$ will not be destroyed by small $\mathscr{C}^{1}$-perturbations. Theorem 1.3 applies, and by combining it with Proposition 5.2 the claim is evident.

5.2. Equality in Corollaries 1.7, 1.8, 4.1 and 4.3, If one substitutes in the formula (4.2) a function for which $\mathscr{B}_{\Omega}^{p}[f]=\mathscr{B}_{\Omega}^{2}[f]$, one acquires the equality in the $\mathscr{L} \log \mathscr{L}$-inequality of Corollary 1.7. Especially, by (5.8) we obtain

Lemma 5.4. Let $\Omega$ be a bounded domain in the plane. If $f$ belongs to the class $\bigcup_{p>2} \mathscr{A}^{p}(\Omega)$, then there is equality in (1.10) in Corollary 1.7 .

Actually, one checks that in the construction, condition (5.5) can be replaced by the analogue $\rho(t)=o\left(\log (1 / t)^{-1}\right)$. Concerning Corollary 4.3, an alternative route to its integral estimates comes by taking the derivative $\partial_{p} \mathscr{B}_{\Omega}^{p}[f]$ at $p=0$. Thus elements of $\bigcup_{p<0} \mathscr{A}^{p}(\Omega)$ give the identity at (4.5).

We next turn our attention to Corollary 1.8. It turns out that there, as well, one has a very extensive class of functions $\mu$ of radial type that yield an equality in the estimates. These functions can be viewed as infinitesimal generators of the expanding class of radial mappings defined above. 
Lemma 5.5. Let $\alpha:(0,1) \rightarrow[0,1]$ be measurable and with the property

$$
\int_{0}^{1} \frac{1-\alpha(t)}{t} \mathrm{~d} t=\infty
$$

Set

$$
\mu(z)=-\frac{z}{\bar{z}} \alpha(|z|) \quad \text { for }|z|<1, \quad \mu(z)=0 \quad \text { for }|z| \geqslant 1 .
$$

Then there is equality in Corollary 1.8, i.e.

$$
\int_{\mathbb{D}}(1-|\mu(z)|) e^{|\mu(z)|}|\exp (\mathbf{S} \mu(z))| \mathrm{d} z=\pi .
$$

Proof. Let $\phi(z)=2 z \int_{|z|}^{1} \frac{\alpha(t)}{t} \mathrm{~d} t$ for $|z|<1$ and set $\phi(z)=0$ elsewhere. Then we compute that $\phi \in \mathscr{W}^{1,2}(\mathbb{C})$ with

$$
\phi_{\bar{z}} \equiv \mu, \quad \phi_{z}=\mathbf{S} \mu(z)=2 \int_{|z|}^{1} \frac{\alpha(t)}{t} d t-\alpha(|z|), \quad|z|<1 .
$$

Thus

$$
\begin{aligned}
& \int_{\mathbb{D}}(1-|\mu(z)|) e^{|\mu(z)|+\operatorname{Re} \mathbf{S} \mu(z)} \mathrm{d} m \\
& =2 \pi \int_{0}^{1}(1-\alpha(t)) \exp \left[2 \int_{t}^{1} \frac{\alpha(s)}{s} d s\right] t \mathrm{~d} t=\pi,
\end{aligned}
$$

as we have the identity

$$
\frac{\mathrm{d}}{\mathrm{d} t}\left(t^{2} \exp \left[2 \int_{t}^{1} \frac{\alpha(s)}{s} \mathrm{~d} s\right]\right)=2 t(1-\alpha(t)) \exp \left[2 \int_{t}^{1} \frac{\alpha(s)}{s} \mathrm{~d} s\right]
$$

and our assumption gets rid of the substitution at $t=0$.

More complicated examples may be obtained by a similar iteration procedure as described above.

Finally, equality in (4.1) obviously implies that necessarily the distortion function $K(z, f) \equiv K$ in $\Omega$. Hence examples are produced by specific functions $\rho$ in (5.1), the powers

$$
\rho_{K}(t)=R^{1-1 / K} t^{1 / K}, \quad r<t<R,
$$

where $\rho_{K}(t)$ is linear on $(0, r]$, if $r>0$. For $2 \leqslant p<\frac{2 K}{K-1}$, let $\mathscr{A}_{K}^{p}(\Omega)$ denote the subclass of $\mathscr{A}^{p}(\Omega)$ consisting of those piecewise radial mappings where, first, we fill the domain $\Omega$ by discs or annuli up to measure zero, second, at each construction step choose $\rho=\rho_{K}$, and third, choose $r=0$ at any possible subdisk remaining in the limiting packing construction. This ensures that the limiting function $f$ does not remain linear in any subdisk, so that we have $K(z, f) \equiv K$ up to a set of measure zero. Then, as $|\Omega|<\infty$, it is easy to see that convergence $f_{\infty}=\lim _{j \rightarrow \infty} f_{j}$ takes place in $\mathscr{W}^{1, p}$ since now $1-p\left|\mu_{j}(z)\right|\left(1+\left|\mu_{j}(z)\right|\right)^{-1} \geqslant c_{0}>0$. Moreover, since there is equality in Theorem 3.5 and $K(z, f) \equiv K$, one obtains for any $f \in \mathscr{A}_{K}^{p}(\Omega)$ that

$$
\frac{1}{|\Omega|} \int_{\Omega}|D f(z)|^{p} \mathrm{~d} z=\frac{2 K}{2 K-p(K-1)}
$$


5.3. On $\mathscr{L}^{p}$-bounds for quasiconformal mappings in $\mathbb{R}^{n}$. Let us close with a discussion on higher dimensional analogues of our optimal results in the plane. This leads to new conjectures on the $\mathscr{L}^{p}$-regularity problem of quasiconformal maps in space. We begin with the following statement in all dimensions.

Theorem 5.6. The functions $\boldsymbol{B}_{p}^{n}: \mathbb{R}^{n \times n} \rightarrow \mathbb{R}$ defined by

$$
\boldsymbol{B}_{p}^{n}(A)=\left(\frac{p}{n} \operatorname{det} A+\left(1-\frac{p}{n}\right)|A|^{n}\right) \cdot|A|^{p-n}, \quad p \geqslant n,
$$

are rank-one concave.

For the proof and more information on this topic, see [32. Of course, one may be tempted to show that $\mathbf{B}_{p}^{n}$ is quasiconcave, but that is beyond our reach in such a generality. However, we state here potential consequences of the conceivable quasiconcavity of the above $n$-dimensional Burkholder functional for quasiconformal maps, i.e. analogues of our optimal results in the plane.

Conjecture 5.7. Suppose $f: \mathbb{B} \rightarrow \mathbb{B}$ is a $K$-quasiconformal mapping of the unit ball onto itself that is equal to the identity on $\partial \mathbb{B}$. Then,

$$
f_{\mathbb{B}}\left(n-p+\frac{p}{K(x)}\right)|D f(x)|^{p} \mathrm{~d} x \leqslant n, \quad \text { for } n \leqslant p \leqslant \frac{n K}{K-1} .
$$

Therefore,

$$
f_{\mathbb{B}}|D f(x)|^{p} \mathrm{~d} x \leqslant \frac{n K}{n K-p(K-1)} .
$$

There are also sharp estimates with the critical upper exponent $p=\frac{n K}{K-1}$,

$$
f_{\mathbb{B}}\left(\frac{1}{K(x)}-\frac{1}{K}\right)|D f(x)|^{\frac{n K}{K-1}} \mathrm{~d} x \leqslant 1-\frac{1}{K} .
$$

Hence

$$
\int_{\mathbb{E}}|D f(x)|^{\frac{n K}{K-1}} \mathrm{~d} x \leqslant|\mathbb{B}|, \quad \text { where } \mathbb{E}=\{x \in \mathbb{B} ; K(x)=1\} .
$$

All the above inequalities are sharp; $n$-dimensional radial and power mappings produce examples for the equalities.

\section{ACKNOWLEDGEMENTS}

The first author was supported by the Academy of Finland grant 1134757 and by the EU-network CODY. The second author was supported by the NSF grant DMS-0800416 and the Academy of Finland grant 1128331. The third author was supported by projects 1134757 and 1138896 of the Academy of Finland and by the Swiss NSF. Each of the authors was supported by the Academy of Finland CoE in Analysis and Dynamics research, grant 1118634.

Part of the research took place when the first author was visiting UAM and ICMAT at Madrid and MSRI at Berkeley. The first author thanks both institutes for the inspiring atmosphere and warm hospitality. 


\section{REFERENCES}

1. L. Ahlfors, Lectures on quasiconformal mappings. Second edition. With supplemental chapters by C. J. Earle, I. Kra, M. Shishikura and J. H. Hubbard. University Lecture Series, 38. American Mathematical Society, Providence, RI, 2006. MR.2241787 (2009d:30001)

2. L. Ahlfors, L. Bers, Riemann's mapping theorem for variable metrics. Ann. of Math. (2) 72 (1960) 385-404. MR0115006 (22:5813)

3. S.S. Antman, Fundamental mathematical problems in the theory of nonlinear elasticity, Numerical solution of partial differential equations, III, Academic Press, New York, 1976, pp. 35-54. MR0468503 (57:8335)

4. K. Astala, Area distortion of quasiconformal mappings. Acta Math. 173 (1994), no. 1, 37-60. MR:1294669 (95m:30028b)

5. K. Astala, T. Iwaniec, G. J. Martin, Elliptic partial differential equations and quasiconformal mappings in the plane, Princeton University Press, 2009. MR2472875 (2010j:30040)

6. K. Astala, T. Iwaniec, G. J. Martin, J. Onninen, Extremal mappings of finite distortion. Proc. London Math. Soc. (3) 91 (2005), 655-702. MR2180459 (2006h:30016)

7. K. Astala, V. Nesi, Composites and quasiconformal mappings: new optimal bounds in two dimensions. Calc. Var. Partial Differential Equations 18 (2003), no. 4, 335-355. MR2020365 (2005d:74035)

8. A. Baernstein, S. Montgomery-Smith, Some conjectures about integral means of $\partial f$ and $\bar{\partial} f$. Complex analysis and differential equations (Uppsala, 1997), 92-109, Acta Univ. Upsaliensis Skr. Uppsala Univ. C Organ. Hist., 64, Uppsala Univ., Uppsala, 1999. MR.1758918 (2001i:30002)

9. J. Ball, Convexity conditions and existence theorems in non-linear elasticity. Arch. Ration. Mech. Anal., 63(1977), 337-403. MR0475169 (57:14788)

10. J. M. Ball, Constitutive inequalities and existence theorems in nonlinear elastostatics, Nonlinear analysis and mechanics: Heriot-Watt Symposium (Edinburgh, 1976), Vol. I, pp. 187-241. Res. Notes in Math., No. 17, Pitman, London, 1977. MR0478899 (57:18357)

11. J.M. Ball, Does rank-one convexity imply quasiconvexity? In Metastability and Incompletely Posed Problems, volume 3, pages 17-32. IMA volumes in Mathematics and its Applications, 1987. MR870007 (88c:49009)

12. J.M. Ball, Sets of gradients with no rank-one connections. J. Math. Pures Appl. (9) 69 (1990), no. 3, 241-259. MR1070479 (91k:49008)

13. J. Ball, The calculus of variations and material science, Current and Future Challenges in the Applications of Mathematics, Providence, RI, 1997. Quart. Appl. Math., 56 (1998), 719-740. MR1668735 (2000i:49002)

14. R. Bañuelos, The foundational inequalities of D.L. Burkholder and some of their ramifications, Dedicated to Don Burkholder, preprint, 2010.

15. R. Bañuelos, P. Janakiraman, $L^{p}$-bounds for the Beurling-Ahlfors transform. Trans. Amer. Math. Soc. 360 (2008), no. 7, 3603-3612. MR2386238(2009d:42032)

16. R. Bañuelos, A. Lindeman, A martingale study of the Beurling-Ahlfors transform in $R^{n}$. J. Funct. Anal. 145 (1997), no. 1, 224-265. MR1442167 (98a:30007)

17. R. Bañuelos, G. Wang, Sharp inequalities for martingales with applications to the BeurlingAhlfors and Riesz transforms. Duke Math. J. 80 (1995), no. 3, 575-600. MR.1370109 (96k:60108)

18. B.V. Bojarski, Homeomorphic solutions of Beltrami systems. (Russian) Dokl. Akad. Nauk SSSR (N.S.) 102 (1955), 661-664. MR0071620 (17:157a)

19. B.V. Bojarski, Generalized solutions of a system of differential equations of the first order and elliptic type with discontinuous coefficients. Translated from the 1957 Russian original. With a foreword by Eero Saksman. Report 118. University of Jyväskylä, Department of Mathematics and Statistics, 2009. iv+64 pp. MR2488720 (2010j:30096)

20. D.L. Burkholder, An elementary proof of an inequality of R. E. A. C. Paley, Bull. London Math. Soc. 17 (1985), 474-478. MR806015 (87a:42041)

21. D.L. Burkholder, A sharp and strict $L^{p}$-inequality for stochastic integrals. Ann. Probab. 15 (1987), no. 1, 268-273. MR877602 (88d:60156)

22. D.L. Burkholder, A proof of Petczyński's conjecture for the Haar system, Studia Math. 91 (1988), 79-83. MR957287 (89j:46026) 
23. D.L. Burkholder, Sharp inequalities for martingales and stochastic integrals. Colloque Paul Lévy sur les Processus Stochastiques (Palaiseau, 1987). Astérisque No. 157-158 (1988), 75-94. MR976214 (90b:60051)

24. P.G. Ciarlet, Mathematical Elasticity, I, North-Holland, Amsterdam, 1987. MR0936420 (89e:73001)

25. O. Dragicevic, A. Volberg, Sharp estimate of the Ahlfors-Beurling operator via averaging martingale transforms. Michigan Math. J. 51 (2003), no. 2, 415-435. MR1992955 (2004c:42030)

26. D. Faraco, L. Székelyhidi, Tartar's conjecture and localization of the quasiconvex hull in $\mathbb{R}^{2 \times 2}$. Acta Math. 200 (2008), no. 2, 279-305. MR2413136 (2009j:30104)

27. S. Geiss, S. Montgomery-Smith, E. Saksman, On singular integral and martingale transforms. Trans. Amer. Math. Soc. 362 (2010), no. 2, 553-575. MR2551497 (2011a:60168)

28. L. Greco, L. T. Iwaniec, New inequalities for the Jacobian. Ann. Inst. H. Poincaré Anal. Non Linéaire 11 (1994), no. 1, 17-35. MR1259100 (95b:42020)

29. S. Hencl, P. Koskela, J. Onninen, A note on extremal mappings of finite distortion, Math. Res. Lett. 12 (2005), no. 2-3, 231-239. MR2150879 (2006a:30022)

30. O. Lehto, K.I. Virtanen, Quasiconformal mappings in the plane. Second edition. Translated from the German by K. W. Lucas. Die Grundlehren der mathematischen Wissenschaften, Band 126. Springer-Verlag, New York-Heidelberg, 1973. viii+258 pp. MR0344463(49:9202)

31. T. Iwaniec, Extremal inequalities in Sobolev spaces and quasiconformal mappings. Z. Anal. Anwendungen 1 (1982), no. 6, 1-16. MR719167 (85g:30027)

32. T. Iwaniec, Nonlinear Cauchy-Riemann operators in $\mathbb{R}^{n}$. Trans. Amer. Math. Soc. 354 (2002), no. 5, 1961-1995. MR1881026(2003i:35092)

33. T. Iwaniec, L. Kovalev, J. Onninen, Diffeomorphic approximation of Sobolev homeomorphisms. Arch. Rat. Mech. Anal., to appear.

34. T. Iwaniec, G. Martin, Geometric function theory and non-linear analysis. Oxford Mathematical Monographs. The Clarendon Press, Oxford University Press, New York, 2001. xvi+552 pp. MR 1859913(2003c:30001)

35. P. Koskela, J. Onninen, Mappings of finite distortion: decay of the Jacobian in the plane, Adv. Calc. Var. 1 (2008), no. 3, 309-321. MR2458240 (2009j:30049)

36. R. Mañé, P. Sad, D. Sullivan, On the dynamics of rational maps. Ann. Sci. École Norm. Sup. (4) 16 (1983), 193-217. MR732343 (85j:58089)

37. C.B. Morrey, Quasi-convexity and the lower semicontinuity of multiple integrals, Pacific J. Math., 2 (1952), 25-53. MR.0054865 (14:992a)

38. C.B. Morrey, Multiple integrals in the calculus of variations. Springer-Verlag, New York, 1966. MR.0202511 (34:2380)

39. S. Müller, A surprising higher integrability property of mappings with positive determinant, Bull. Amer. Math. Soc., 21 (1989), 245-248. MR999618 (90c:49022)

40. S. Müller, Rank-one convexity implies quasiconvexity on diagonal matrices, Internat. Math. Res. Notices 1999, no. 20, 1087-1095. MR1728018 (2001f:49030)

41. F. Nazarov, S. Treil, A. Volberg, The Bellman functions and two-weight inequalities for Haar multipliers, J. Amer. Math. Soc. 4 (1999), 909-928. MR.1685781 (2000k:42009)

42. F. Nazarov, S. Treil, A. Volberg, Bellman function in stochastic control and harmonic analysis. Systems, approximation, singular integral operators, and related topics (Bordeaux, 2000), 393-423, Oper. Theory Adv. Appl., 129, Birkhäuser, Basel, 2001. MR1882704 (2003b:49024)

43. P. Pedregal, V. Šverák, A note on quasiconvexity and rank-one convexity for $2 \times 2$ matrices. J. Convex Anal. 5 (1998), no. 1, 107-117. MR.1649433 (99i:49005)

44. S. Petermichl, A. Volberg, Heating of the Ahlfors-Beurling operator: weakly quasiregular maps on the plane are quasiregular. Duke Math. J. 112 (2002), no. 2, 281-305. MR.1894362 (2003d:42025)

45. W. Rudin, Functional analysis, 2nd edition, McGraw-Hill, 1990. MR.1157815 (92k:46001)

46. V. Šverák, Rank-one convexity does not imply quasiconvexity, Proc. Roy. Soc. Edinburgh Sect. A 120 (1992), no. 1-2, 185-189. MR.1149994 (93b:49026)

47. A. Volberg, F. Nazarov, Heat extension of the Beurling operator and estimates for its norm. (Russian) Algebra i Analiz 15 (2003), no. 4, 142-158; translation in St. Petersburg Math. J. 15 (2004), no. 4, 563-573. MR2068982(2005f:30042) 
Department of Mathematics and Statistics, University of Helsinki, P. O. Box 68, Gustaf Hällströmin katu 2B, FI-00014, Helsinki, Finland

E-mail address: kari.astala@helsinki.fi

Department of Mathematics, Syracuse University, Syracuse, New York 13244, USA, and Department of Mathematics and Statistics, University of Helsinki, P. O. Box 68, Gustaf Hällströmin KATU 2B, FI-00014, Helsinki, Finland

E-mail address: tiwaniec@syr.edu

Department of Mathematics and Statistics, University of Helsinki, P. O. Box 68, Gustaf Hällströmin katu 2B, FI-00014, Helsinki, Finland

E-mail address: istvan.prause@helsinki.fi

Department of Mathematics and Statistics, University of Helsinki, P. O. Box 68, Gustaf Hällströmin KAtu 2B, FI-00014, Helsinki, Finland

E-mail address: eero.saksman@helsinki.fi 